\title{
Laparoscopic management of adnexal masses in admitted patients at tertiary care centre, Bhilai, Chhattisgarh, India
}

\section{Meena Naik*, Pooja Singh, Rekha Ratnani}

Department of Obstetrics and Gynaecology, Shri Shankaracharya Institute of Medical Sciences, Bhilai, Chhattisgarh, India

Received: 30 May 2021

Accepted: 30 June 2021

\section{*Correspondence:}

Dr. Meena Naik,

E-mail: m.naik1971@yahoo.com

Copyright: (C) the author(s), publisher and licensee Medip Academy. This is an open-access article distributed under the terms of the Creative Commons Attribution Non-Commercial License, which permits unrestricted non-commercial use, distribution, and reproduction in any medium, provided the original work is properly cited.

\section{ABSTRACT}

Background: Laparoscopy is most widely accepted procedure in gynaecological cases both for diagnostic and operative purposes. It is associated with early recovery, shorter hospital stay and significant patient satisfaction. This study has been conducted to evaluate the effectiveness and safety of laparoscopy in the management of adnexal masses. Our Primary aim was to find out indications, intraoperative findings and different interventions to deal with adnexal mass. The objective of the study was to determine laparoscopic management of adnexal mass in all women attending Obstetrics and Gynaecology department, Shanakaracharya Institute of Medical Science, Bhilai, Chhattisgarh.

Methods: This is a retrospective record-based observational study conducted over a period of one year from March 2020 to March 2021 after getting approval from the institutional ethical committee. Patients diagnosed with adnexal mass were evaluated and data was collected and analyzed.

Results: Of the 130 cases of adnexal mass admitted in the hospital during the period under review, 43 cases (33\%) were diagnosed as ovarian cyst among which simple cystic ovarian cyst was found to be most common etiology followed by ectopic pregnancy (41 cases). Majority of cases were managed laparoscopically with a good success rate, better outcome and short hospital stay. Conversion rate to laparotomy was $3.8 \%$ mainly seen in cases with complex ovarian mass or dense adhesions.

Conclusions: Diagnosis of adnexal mass is important and it may affect females of any age group. Laparoscopy is a better approach to deal with adnexal masses (except malignancy) as it has better paronamic vision with less complications, less post-operative pain, shorter hospital stay. Careful patient selection should also be taken into account. Current study is an overview in favor of laparoscopic management of adnexal mass.

Keywords: Adnexal mass, Ovarian cyst, Ectopic pregnancy, Laparoscopy

\section{INTRODUCTION}

Over years, laparoscopy has evolved from a limited gynecological surgical procedures to a major surgical tool used for management of a variety of gynecological conditions. Recently, laparoscopy has emerged as one of the most common surgical procedures. The faster recovery time, minimal pain, adhesion prevention, shorter hospital stay and better aesthetic results has made laparoscopy immensely popular. Also, technical parameters such as the magnified view during the procedure and relatively small risk of complications resulted in the wide use of laparoscopic surgery in gynecology. Laparoscopy has now become the gold standard method for management of a wide range of gynecological ailments, including the adnexal masses.

During the last decades laparoscopic treatment has been established as a routine method of benign ovarian masses. However, it remains controversial for which cases the method is indicated. Although, most of the adnexal masses arise from ovaries, a wide variety of pathologies may be 
associated. Tuboovarian mass/abscess, ectopic pregnancy, appendicular mass etc are the common pathologies that need to be differentiated. ${ }^{1}$ A number of non-neoplastic and neoplastic and lesions occur within the ovaries. Most are non-neoplastic are functional in nature and resolve with minimal treatment. However, some may herald an underlying malignant process. When cysts are large, persistent, or painful, surgery may be required. ${ }^{2}$

According to the current evidence the parameters which should be examined preoperatively in order to exclude the possibility of a malignancy are the following: morphology of the ovarian cyst (septations internal bordered, papillary projections, echogenicities, and volume). Doppler ultrasonography could also be helpful by examining the mass vascularisation, which increases the possibility of a malignancy. The sonological detection of ovarian malignancy is quite good and acceptable. The predictive value is about $96 \%$ in detection of the benign masses. ${ }^{3}$ In addition elevated tumor markers like CA 19-9, CA 125 , and tumor associated trypsin inhibitior (TATI) increase our preoperative diagnostic aims. Magnetic resonance imaging (MRI) increases the specificity of imaging evaluation for adnexal masses, especially when they are indeterminate on ultrasound. ${ }^{4}$ MRI has a high accuracy in differentiating benign from malignant masses. Endometriomas, Teratomas, simple cysts, extrauterine fibroids, and hydrosalpinges can be diagnosed with high specificity. The procedure, being non-invasive, is widely acceptable for establishing the diagnosis.

\section{METHODS}

The study was retrospective record-based study. The place of study was Shri Shankaracharya Institute of Medical Sciences, Bhilai. The study duration was March 2020 March 2021.

\section{Inclusion criteria}

Benign mass (USG/MRI report). Preoperative estimation of the tumor markers levels especially CA 125 (normal range $\quad 0-35 \mathrm{mU} / \mathrm{L})$. Ruptured/unruptured ectopic pregnancy. Haemodynamically stable patient.

\section{Exclusion criteria}

Contraindications for laparoscopy, such as high BMI or medical reasons. Ultrasonographic evidence of malignancy. Exceptionally high preoperative $\mathrm{Ca} 125$ values (more than $200 \mathrm{U} / \mathrm{ml}$ ). Patient is in shock/haemodynamically unstable.

\section{Procedure}

Patients who fulfilled the above inclusion criteria were included in the study. During this period, 130 patients underwent laparoscopy for various types of adnexal masses. Data collection was done from the records maintained by hospital after getting approval from the institutional ethical committee. Cases were operated on elective/emergency basis. Detailed history, a thorough general physical examination and pre-anesthetic work-up was done on all patients. Trans-vaginal ultrasound scanning was used as primary imaging and preoperative tumor markers were checked.

Large (more than $12 \times 12 \mathrm{~cm}$ ) solid, fixed or irregular adnexal masses suspicious of malignancy were treated by laparotomy.

After written informed consents, all the patients fulfilling inclusion criteria were taken for the procedures under general anesthesia. Preoperative findings were noted. Assessment of indications, intraoperative findings and various interventions done during the procedures were carefully analyzed. All the specimens were sent for histopathological examinations post operatively. All the patients received $\mathrm{i} / \mathrm{v}$ antibiotics preoperatively followed by oral antibiotics

\section{Data analysis}

Datas were recorded in Excel sheet and descriptive analysis was performed and results were expressed in numbers and percentages.

\section{RESULTS}

According to the statistics, 130 patients underwent laparoscopic surgery for management of adnexal mass. 96.2\% patients underwent successful laparoscopic surgeries while conversion to laparotomy was $3.8 \%$. The age group considered was from 13 years to 55 years.

Table 1: Histopathology of adnexal mass.

\begin{tabular}{|lll|}
\hline Histopathology & $\begin{array}{l}\text { Number } \\
\text { of cases }\end{array}$ & Percentage \\
\hline Ectopic pregnancy & 41 & $31.5 \%$ \\
\hline $\begin{array}{l}\text { Endometrioma } \\
\text { (chocolate cyst) }\end{array}$ & 16 & $12.3 \%$ \\
\hline Ovarian cyst (benign) & 43 & $33 \%$ \\
\hline Hydrosalpinx & 3 & $2 \%$ \\
\hline Tubo-ovarian mass & 9 & $6.9 \%$ \\
\hline Ovarian torsion & 15 & $11.5 \%$ \\
\hline
\end{tabular}

In all cases the diagnosis was confirmed histologically. According to the results, benign ovarian cyst was most common disease, diagnosed in 43 (33\%) of the cases, among which serous cystadenoma was found to be most common, followed by ectopic pregnancy, being the 2 nd most common cause (31.5\%), followed by Endometrioma, contributing to $12.3 \%$. It was found most commonly in women between 25-35 years age groups. Whereas the diagnosis of the rest of the masses was as follows: Ovarian torsion most commonly seen in young girls contributing to $11.5 \%$, tubo-ovarian mass was diagnosed in 15 patients $(6.9 \%)$, hydrosalpinx in 3 cases $(2 \%)$. (Table 1, Figure 1) 


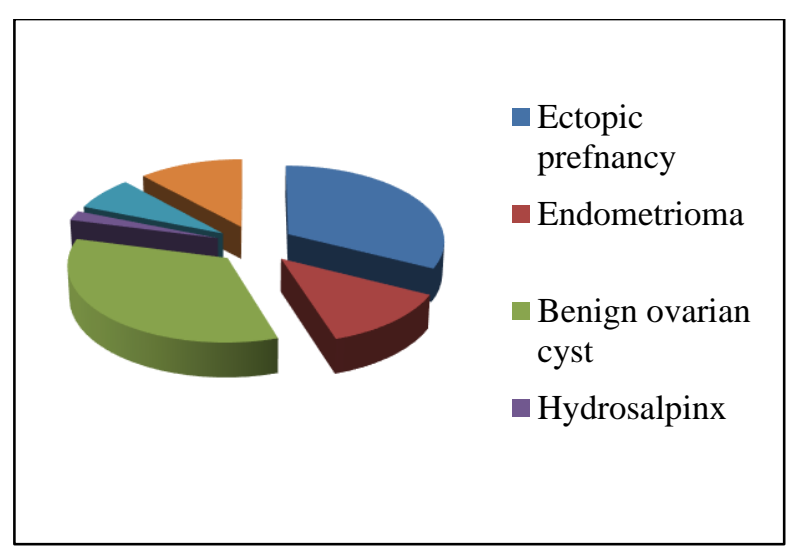

Figure 1: Histopathology of adnexal mass.

Table 2: Types of operations for adnexal mass.

\begin{tabular}{|lll|}
\hline Surgical procedure & $\begin{array}{l}\text { Number } \\
\text { of cases }\end{array}$ & $\begin{array}{l}\text { Percentage } \\
\text { Ovarian cystectomy }\end{array}$ \\
51 & $39.2 \%$ \\
\hline Salpingectomy & 34 & $26.1 \%$ \\
\hline Oopherectomy & 6 & $4.6 \%$ \\
\hline Salpingec-oopherectomy & 17 & $13.0 \%$ \\
\hline Salpingostomy & 5 & $3.8 \%$ \\
\hline Adhesiolysis+cystectomy & 4 & $3.0 \%$ \\
\hline $\begin{array}{l}\text { Excision of paraovarian } \\
\text { mass }\end{array}$ & 1 & $0.7 \%$ \\
\hline Cyst aspiration & 10 & $7.69 \%$ \\
\hline $\begin{array}{l}\text { Conversion to } \\
\text { laparotomy }\end{array}$ & 5 & $3.8 \%$ \\
\hline
\end{tabular}

Most common surgery performed was cystectomy. Ovarian cystectomy is the method of choice in young patients to preserve fertility, as most of our patients were in reproductive age group and have presented as infertility. In some cases, adhesiolysis was done along with cystectomy.

Cyst aspiration was also tried in one case as ovary preserving procedure.

All cases of hydrosalpinx, where tube was infected and non-functional, salpingectomy was done.

Conservative surgeries like salpingostomy was done for unruptured ectopic pregnancy and young females in order to preserve fertility, while Salpingectomy was done in for ruptured tubal ectopic pregnancy as standard procedure.

In cases where ovary was twisted and cyst with tubes were suspected to be necrosed/ gangrenous, SalpingoOopherectomy was performed.

In 5 cases $(11.5 \%)$ laparoscopy was converted to laparotomy due to technical difficulties, suspicion of cancer, severe adhesions and due to bleeding that could not be controlled safely by laparoscopy (Table 2, Figure 2).

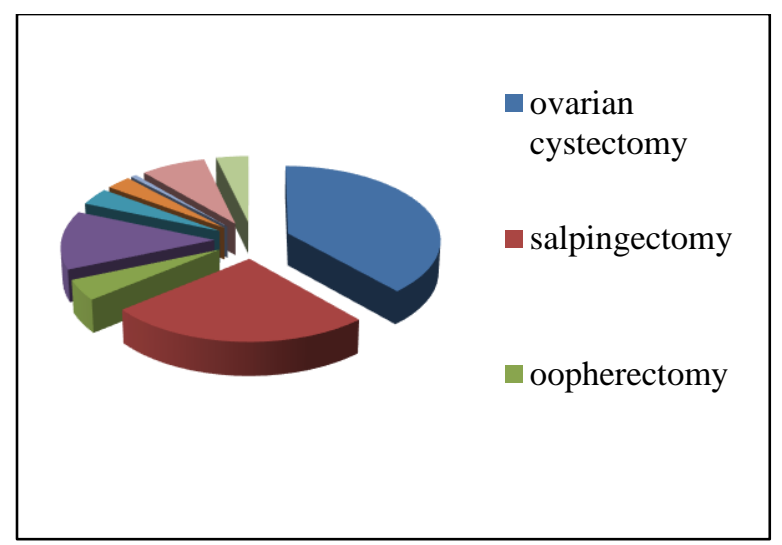

Figure 2: Types of operations performed.

There were no major complications noted in this study. The most common complication was post-operative fever (7.6\%), which was managed by IV antibiotics and various tests (blood culture, urine c/s, high vaginal swab). Intraoperative bleeding was 2nd most common complication seen among $2.3 \%$ of the cases, managed by intra-operative blood transfusion. There were no bowel, bladder injury noted in this cases. Port site infection was minimal, seen among $1.5 \%$ of cases. Average hospital stay was found to be 4-5days and was seen to be prolonged in some cases. (Table 3)

Table 3: Complications.

\begin{tabular}{|lll|}
\hline Types of complication & $\begin{array}{l}\text { Number } \\
\text { of cases }\end{array}$ & Percentage \\
\hline Post-operative fever & 10 & $7.6 \%$ \\
\hline $\begin{array}{l}\text { Intra-operative } \\
\text { bleeding }\end{array}$ & 3 & $2.3 \%$ \\
\hline Bowel/bladder injury & 0 & $0 \%$ \\
\hline Port site infection & 2 & $1.5 \%$ \\
\hline Average hospital stay & $4-5$ days & \\
\hline
\end{tabular}

\section{DISCUSSION}

Benign ovarian masses consist of one of the most common issues which the gynecologist has to treat. Laparotomy was considered to be the indicated treatment method until endoscopic methods has been developed years ago. Since this time the continual development of laparoscopy allowed us today to accept endoscopy as a safe and effective alternative to laparotomy, which has additionally important advantages concerning the patient's condition after surgery. Even if this successful treatment tends to be the most frequent used surgical method, there are still debates concerning the safety and the effectiveness especially for the endometriosis cases or suspicious masses. A meta-analysis of related studies comparing laparoscopy to laparotomy in treatment of benign adnexal masses points out the advantages of this proceed. ${ }^{5}$ It has been shown that laparoscopy caused less adverse effects, less postoperative pain, shorter stay in hospital, smaller readmission rates, better panoramic vision, and 
significantly lower cost. It is today almost accepted that laparoscopy is more beneficial than laparotomy but the importance of patient selection and availability of a gynecologic oncologist should be taken into account.

Grammatikakis et al did a large series study on laparoscopic management of adnexal masses in 1552 women. ${ }^{4}$ Most common pathology detected was endometrioisis $(80.6 \%) .11 .5 \%$ of cases were converted into laprotomy due to dense adhesions, technical difficulties or suspicion of malignancy. As for complications, major complication occurred in very small number of patients $(0.6 \%)$ in the form of bowel or bladder injuries.

Bhattacharjee et al did a hospital-based study on Laparoscopic management of adnexal masses. ${ }^{6}$ In their study, most of the patients were from the age-group 25-30 years. Most common indication was diagnosed to be ovarian tumor of varied pathology. Ovarian cystectomy $(41.2 \%)$ was the most commonly performed procedure. Most common histopathological finding was functional ovarian cyst (26.9\%). Only $3.1 \%$ of cases needed conversion to laparotomy for the completion of the procedure. Complication rate was minimal.

Duggal et al did a retrospective study on 121 cases of adnexal masses which were managed laparoscopically. ${ }^{7}$ Most common histology finding was functional cyst in 30 cases. Most common procedure was ovarian cystectomy, done in 76 cases. In one case laparotomy had to be done to complete the procedure.

In our study, 130 patients fulfilling the inclusion criteria underwent laparoscopic management. Ovarian cyst was the major pathology (33\%) followed by ectopic pregnancy $(31.5 \%)$. Ovarian cystectomy was performed as primary procedure $(39.2 \%)$ for ovarian cyst and salpingectomy was done as procedure of choice for ectopic pregnancy (26\%). Histopathology showed simple serous cyst as common finding. Conversion rate from laparoscopy to laparotomy was $3.8 \%$. Post-operative complications were minimal and most commonly it was post-operative fever which subsided by IV antibiotics.

\section{CONCLUSION}

Adnexal mass is an important pathology which may affect females of all age groups. Majority of them are diagnosed incidentally. Some of these may undergo rupture or torsion. They may also pose significant diagnostic and management dilemmas. Laparoscopy is safe approach and can be used in managing patients with benign adnexal masses. Proper selection of cases is important and can be achieved by ultrasonography, CA-125, CT scan and MRI. Our study confirms that laparoscopy can be safely performed for management of benign adnexal masses after proper selection of cases by using ultrasonography, MRI and tumor markers like CA-125 as primary investigations. We recommend operative laparoscopy as the gold standard for the surgical treatment of the ovarian cysts, Torsion or unruptured ectopic pregnancy. It is a choice of surgery as it is associated with reduced operative blood loss, fewer postoperative complications, shorter hospitalization, less pain and earlier recovery as compared with laparotomy. Also it is the procedure of choice for fertility conserving/enhancing surgeries. Laparoscopic surgery seems to offer significant advantages such as reduced hospital stay, less adverse effects, better quality of life, and superior vision especially on surgical treatment of cases like endometriosis. Proper selection of cases, adequate surgical skills along with multidisciplinary team approach are imperative for good patient outcome.

\section{Funding: No funding sources \\ Conflict of interest: None declared}

Ethical approval: The study was approved by the Institutional Ethics Committee

\section{REFERENCES}

1. Zaman S, Majid S, Hussain M, Chughtai O, Mahboob J, Chughtai S. A retrospective study of ovarian tumours and tumour-like lesions. Journal of Ayub Medical College Abbottabad. 2010;22(1):104-8.

2. Matsushita H, Watanabe K, Yokoi T, Wakatsuki A. Unexpected ovarian malignancy following laparoscopic excision of adnexal masses. Human Reproduction. 2014;29(9):1912-7.

3. Karnik A, Tembey RA, Mani S. Value of MRI in characterizing adnexal masses. The Journal of Obstetrics and Gynecology of India. 2015;65(4):259-66.

4. Grammatikakis I, Trompoukis P, Zervoudis S, Mavrelos C, Economides P, Tziortzioti V et al. Laparoscopic treatment of 1522 adnexal masses: an 8-year experience. Diagnostic and therapeutic endoscopy. 2015;2015.

5. Medeiros LR, Stein AT, Fachel J, Garry R, Furness S. Laparoscopy versus laparotomy for benign ovarian tumor: a systematic review and meta-analysis. International Journal of Gynecologic Cancer. $2008 ; 18(3)$.

6. Bhattacharjee S, Sharma S. Laparoscopic management of adnexal masses: a hospital-based study. International Journal of Reproduction, Contraception, Obstetrics and Gynecology. 2008;7(4):1428.

7. Duggal BS, Tarneja P, Sharma RK, Rath SK, VSM RW. Laparoscopic management of adnexal masses. Medical Journal Armed Forces India. 2004;60(1):28-30.

Cite this article as: Naik M, Singh P, Ratnani R. Laparoscopic management of adnexal mass in admitted patients at tertiary care centre, Bhilai, Chhattisgarh, India. Int J Reprod Contracept Obstet Gynecol 2021;10:3119-22. 